\title{
Research on the Operation and Inspection System of UHV and Cross-Regional Distribution Network Based on Big Data Technology
}

\author{
Ji Zhixiang, Deng Chunyu \\ China Electric Power Research Institute, Haidian District, Beijing, China
}

\begin{abstract}
With the development of UHV and cross-regional distribution network, the operation and inspection of electric transmission and transformation equipment need to use big data and other new technologies to build a smart grid operation and inspection system, in order to better support the operation and inspection business. This paper analyzes the new development situation of UHV and trans-area power grid and the demand of smart grid operation and inspection service under the new situation. Combining big data analysis technology with production command and management practice, based on data and business requirements, this paper puts forward the data architecture of intelligent operation and inspection system according to big data technology, gives the technical framework and technology selection of intelligent operation and inspection system, then analyzes the application framework of intelligent operation and inspection service. The whole architecture of intelligent operation and inspection system is proposed, which provides better support for the development and application of intelligent operation and inspection system through the key architecture design. It has far-reaching application value and broad application prospect.
\end{abstract}

\section{Introduction}

In recent years, with the rapid growth of power network scale, UHV and cross-regional power network equipment is increasing, UHV, large capacity, long distance and trans-area large power grid pattern put forward higher requirements for the operation, inspection, management and capabilities control of electric transmission and transformation equipment.

At present, there are four outstanding problems in the operation and inspection business as follows: First, with the construction of UHV, the interrelation among the power grids are wider and deeper, which makes the original independent operation and inspection network become more relevant in the operation and inspection business, it needs to realize the coordination of interregional power networks operation and inspection through a larger system. Second, the rapid growth of UHV and cross-regional power grid scale leads to the rapid increase of the operating pressure on power grid safety and stability, under such circumstance, field operation and inspection personnel urgently need to enhance the control strength and depth of equipment status, so new technical means need to be used; The third is that the information system has greatly improved the efficiency of operation and inspection business personnel, but with the explosive growth of the data volume of each system, the traditional system architecture can no longer meet the requirements of users for the rapid processing of massive data; Fourth, with the deep integration of operation and inspection work and information system, in addition to the general needs of general functions in the application of the information system, operators often hope to flexibly adjust the functions of the information system according to the actual needs. However, the current information system cannot meet this requirement. Therefore, it necessary to realize the deep integration with the traditional operation and inspection business, build an intelligent operation and inspection system based on big data technology, support the operation and inspection business, change the management mode and work mode of traditional operation and inspection in an all-round way through cloud computing, big data and other new technologies, in order to adapt to the development of smart grid. At present, big data technology has been applied in many fields of power[15].

This paper first analyzes the demand of intelligent operation and inspection service for UHV and trans-area power grid, then researches the service and application characteristics of intelligent operation and inspection platform. Based on big data technology, the data architecture, technical framework and application construction of the layout of power grid operation and

\footnotetext{
* Corresponding author \{jizhixiang,dengchunyu\} @epri.sgcc.com.cna
} 
inspection system are designed, forming the whole framework of intelligent operation and inspection system.

\section{Introduction Of Intelligent Operation And Inspection}

Grid operation and inspection business is mainly responsible for the operation, inspection, inspection, commissioning as well as transformation and replacement of power network equipment. Intelligent operation and inspection technology, which refers to the comprehensive use of big data, cloud computing, Internet of things and other advanced means, with real-time, visual, mobile, interactive and other characteristics, is a generic name of techniques to provide support for the intelligence of equipment, channels, operation, inspection and production management. The intelligent operation and inspection technology is concentrated on improving the automatic perception ability of state information such as equipment and environment, the management and control ability of operation, inspection, repair and other working processes, to construct the professional intelligent system of operation and inspection, thus realizing the deep fusion of "Internet + power grid operation and inspection".

The intelligent operation and inspection system as well as PMS are together used to realize the intelligence of operation and inspection business and production command decision-making. As a basic information platform and business circulation platform, PMS is mainly used for information collection and daily work flow by primary business personnel. As a production command platform, smart operation and inspection system of power grid is mainly used for production command and big data online analysis by the management personnel in charge of the operation and inspection business. The intelligent operation inspection system of power grid is the embodiment of "Internet + power grid operation and inspection" in the aspect of production management intelligentization, so as to improve the control and management of the equipment by the professional managers of operation and inspection at all levels. The intelligent operation and inspection platform shares data source with PMS, to grasp the basic information of operation and inspection more quickly, more accurately, more comprehensively and in a more timely manner by importing the data of PMS into the intelligent operation and inspection system of the power grid. The intelligent management and control of grid operation inspection is mainly divided into two areas: big data analysis and production command and control.

Big data analysis business domain includes equipment state analysis, monitoring and warning, fault evaluation, performance evaluation four business topics. By using the big data processing ability of big data computing framework, the intelligent evaluation of equipment status is analyzed through the whole life cycle. Based on the autonomous learning analysis model, the real-time monitoring and warning of the whole network equipment operating condition is carried out. Integrate various operation data to comprehensively study and judge the fault equipment; Excavate the operation and inspection potential of each unit to evaluate the work performance reasonably. The basis of power grid management and control system is to provide big data computing power for intelligent operation and inspection system.

The production command business domain includes four business topics: process control of operation and inspection, remote consultation, emergency repair and visualization of multiple information. Combined with the presentation ability of GIS and multi-dimensional analysis graphics module, a comprehensive control of the operation and inspection business implementation is carried out with penetration into business processes; Effective integration of the experts resources through breaking geographical space barriers; Connect emergency repair work site to rationally allocate all kinds of operation and inspection resources; Integrate graphics, GIS video and other display means to intuitively display the monitoring of various types of business. To provide visual display for intelligent operation and inspection system is the core of management and control system.

\section{Data Architecture Of Intelligent Operation And Inspection System}

The big data technology and platform can support the grid various tasks very well[6-8]. Intelligent operation and inspection system is built on the basis of big data platform which can support the storage, processing, management and sharing services of all kinds of intelligent operation and inspection business data. The intelligent operation and inspection service data include the main network information data, the real-time data, the running environment data, the equipment condition monitoring data, the inspection and repair data, as well as the operation and inspection management data, such as the production watch data. These data include not only structured data but also unstructured data. Different data, supporting services with great differences, need to be processed according to business requirements and technical characteristics to better support intelligent operation and inspection services. Big data platform has rich interface with various external systems and interactive terminals, supporting the collection and fusion of structured, semi-structured and unstructured data and providing distributed storage, parallel processing, global sharing, application development and other functions. The data architecture is shown in figure 1 .

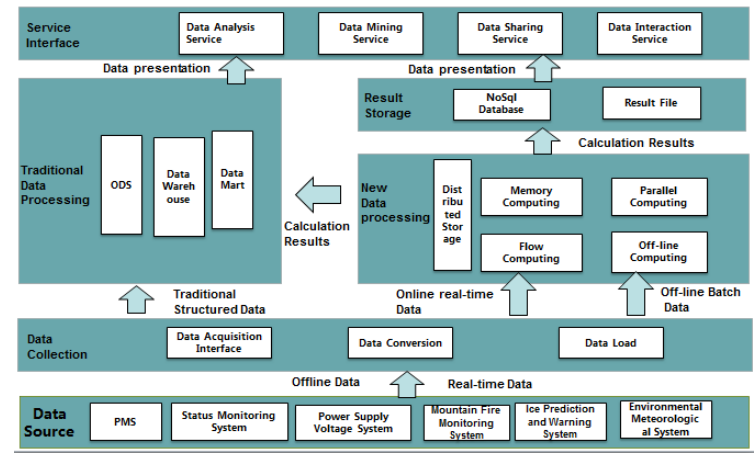


Figure 1. Data Architecture of Intelligent Operation and Insection System

Through batch and real-time collection from the relevant system data sources, together with ETL process in the acquisition layer, operation and inspection data are sent to the traditional or new data processing platform. In the traditional data processing platform and the new data processing platform, the data is stored and processed. ETL process and data processing process are unified by workflow to implement task assembly, scheduling and management. Through the mass computing and analyzing and mining ability of the data, results acquired by the new data processing platform can enter the data mart of the traditional data processing platform. Service and interface layer provides a variety of data services and opens a variety of data interfaces. The external systems/users can access corresponding services according to the authority through the service authentication, the data API and so on.

According to the development and characteristics of the business, the architecture can flexibly compute data or extend the storage resources without affecting the existing business and system architecture.

\section{Technical Framework Of Intelligent Operation And Inspection System}

Intelligent operation and inspection system finds out the laws contained in the data through the collection, storage, calculation of massive data, along with statistical analysis and depth mining. The intelligent operation and inspection system adopts traditional data warehouse and new data processing technology to support on-line, nearline and off-line processing modes. Meanwhile, it utilizes mixed architecture and integrates data warehouse, hadoop, Storm, Mahout and other technical components to achieve unified scheduling and control. And combined with a variety of data analysis models, it achieve big data processing analysis and mining regard to operation and inspection business.

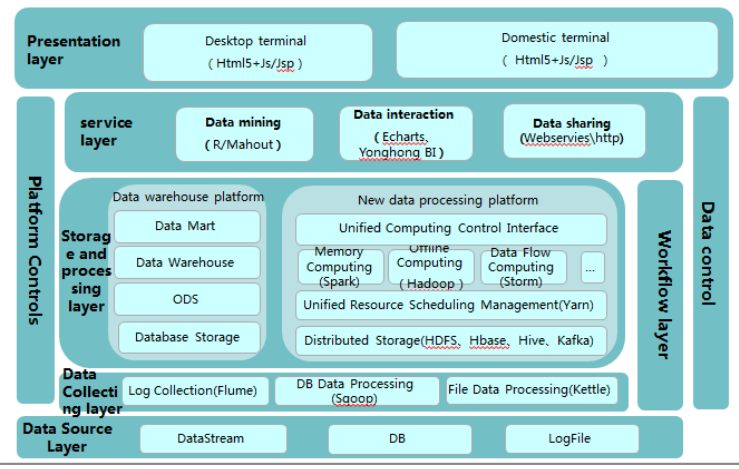

Figure 2. Technical framework diagram

Collection layer: Set up the data collection service cluster with the distributed deployment and linear extended, so as to realize the collection, transformation, loading of the multi-type data. Data acquisition is achieved through the database synchronization tool (Sqoop). Log collection tools can use Flume. Use Kettle to adapts to a variety of data acquisition and processing methods. Data are respectively imported into distributed storage(HDFS, Hbase and Hive) and distributed queue(Kafka) according to the real-time and non-realtime.

Storage and processing layer: The hybrid architecture of traditional data warehouse and new data processing platform is adopted. The new data processing platform adopts the distributed architecture to realize the massive storage of each type of data(structured/semistructured/unstructured data), with data redundancy. The resources can be linearly expanded.

With distributed file system and unified resource scheduling as the core, various computing components are integrated. Distributed architecture is adopted to efficiently integrate offline computing, flow computing, memory computing and other computing frameworks to achieve unified scheduling and control.

Service layer: For the calculation model and specific algorithm, carry out tool and interface graphical encapsulation. The commonly used models of statistics, analysis and mining are encapsulated in a plug-in type so as to reduce the threshold of secondary development. Data analysis services, data mining services, data sharing services and data exchange services are encapsulated into the upper layer, to provide external data capabilities.

Workflow layer: Implementation of unified assembly and scheduling management for various types of tasks(ETL task, computing task, analysis and mining task).

The intelligent operation and inspection system adopts a mixed structure, combines the traditional relational data storage and the new column data storage with the characteristics of the electric power industry. It uses a new data processing technology, which provides an analytical technical service for the application of big data in the intelligent operation and inspection service and supports business applications.

\section{Application Architecture Of Intelligent Operation And Inspection System}

The application architecture of intelligent operation and inspection system is composed of big data platform layer and application layer. Big data platform provides data collection, data storage, data calculation, data analysis, data visualization and so on. Platform management offers cluster management, authority management and security management for intelligent operation and inspection system. Data control mainly realizes metadata management, data audit, data authority management and so on. 


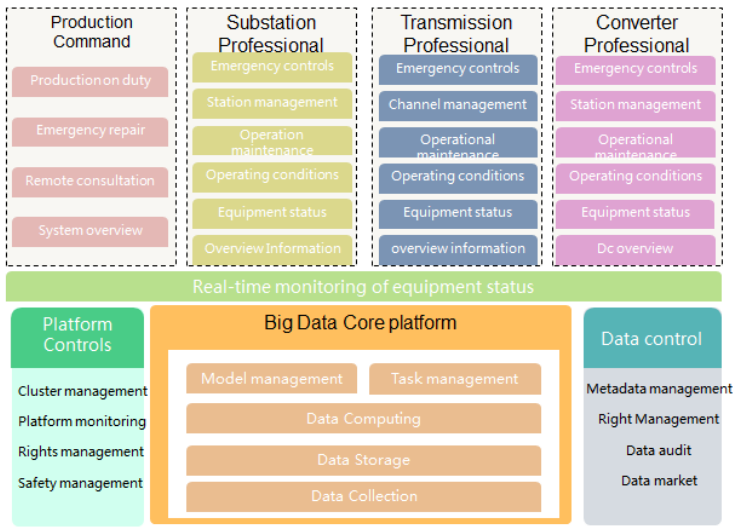

Figure 3. Application architecture of intelligent operation and inspection system

Application layer is the core of intelligent operation and inspection system. Big data application in the intelligent operation and inspection system uses real-time monitoring data based on equipment condition, realizes early warning and state analysis of equipment state through big data. It combines expert knowledge and related factors to realize the function of fault research and judge. Through the real-time monitoring and analysis results of equipment basic data, various applications such as production command, substation, transmission, commutation and so on are supported in the intelligent operation and inspection system.

UHV and transregional power grid operation and inspection system can support "large overhaul" and "large operation" system of large power grid. It can be deployed in related operation and inspection business department of state and provincial power grid to provide UHV and cross-regional grid operation and inspection with business support services, in particular, to supply the UHV AC/DC connected power network and the provincial key grid with a strong interaction and linkage of operational and inspection data.

\section{Summary}

(1) With the development of UHV and trans-area power grid, it is necessary to build UHV and trans-area intelligent operation and inspection system based on big data technology, artificial intelligence and other new technologies, so as to better support the development of operation and inspection business.

(2) The new intelligent operation and inspection system for UHV and cross-regional power grid needs to integrate the traditional data processing architecture and build a new data processing architecture as well according to the data and business needs to improve the data processing capability.

(3) With the development of smart grid and the construction of UHV and cross-region power grid, data interconnection will be formed because of the interconnection of the power grids, therefore, the data will be the core resource. The intelligent operation and inspection system will carry many types of applications of smart grid operation and inspection business, as a result, its fundamental role will become increasingly important.

\section{ACKNOWLEDGEMENTS}

This work is supported by State Grid Research Grant(GYB17201600087).

\section{REFERENCES}

1. Zhang Suxiang, Zhao Binzhen, Wang Fengyu, et al. Short-term power load forecasting based on big data[J]. Proceedings of the CSEE, 2015, 35(1): 3742(in Chinese).

2. Informatization Committee of the CSEE. White paper of electric power big data of China[M]. Beijing: China Electric Power Press, 2013: 10-15(in Chinese).

3. Sun Bolin. Big data technology and its application in power industry[J] . Electric Age, 2013(8) : 18-23(in Chinese).

4. Huang Yanhao, Yu Zhihong, Xie Chang. Study on the application ofelectric power big data technology in power system simulation $[\mathrm{J}]$. Proceedings ofthe CSEE, 2015,35(1): 13-22(in Chinese.)

5. Zhang Dongxia, Miao Xin, Liu Liping,et al. Research on development strategy for smart grid big data[J]. Proceedings of the CSEE, 2015, 35(1): 212(in Chinese)

6. Meng Xiaofeng, Ci Xiang. Big data management: concepts, techniques and challenges[J] . Journal of Computer Research and Development, 2013, 50(1): 146-169(in Chinese).

7. Li Guibing, Luo Hong. Intelligent data analysis under the background of big data[J] . Science \& Technology Information, 2013(30): 11-12(in Chinese).

8. Rao Wei, Ding Jianyong, Lu Qingkai . Cloud computing platform for smart grid[J]. East China Electric Power, 2011, 39(9): 1493-1496. 